\title{
Spectroscopic observations of two proto planetary-nebulae candidates: SS73 56 \& Hen 2-79^
}

\author{
C. B. Pereira, S. J. C. Landaberry, and F. X. de Araújo \\ Observatório Nacional-MCT, Rua José Cristino 77, CEP 20921-400, São Cristóvão, Rio de Janeiro-RJ, Brazil \\ Received 31 October 2002 / Accepted 14 February 2003

\begin{abstract}
We report new spectroscopic observations of two proto planetary-nebulae candidates: SS73 56 and Hen 2-79 between $3200 \AA$ and $8000 \AA$. From the measurements of several forbidden and permitted emission lines of single ionized iron, we derived the color excess and the excitation temperature. We discuss the nature of the two objects based on data obtained. The iron spectrum closely resembles $\eta$ Car but the absence or the weakness of [ $\left.\mathrm{N}_{\text {II }}\right] 5754 \AA$ compared to $\eta$ Car reduces the possibility of being a LBV object. We also compare the two objects with the peculiar emission-line object SS73 11 that was recently analyzed by us.
\end{abstract}

Key words. stars: emission-line, Be - stars: AGB and post-AGB - stars: individual: SS73 56, Hen 2-79

\section{Introduction}

As part of a long-term program to investigate emission-line objects in the southern hemisphere, we are performing a spectroscopic survey at the European Southern Observatory (ESO) of some stars from Sanduleak \& Stephenson (1973) and Weaver (1974) samples. This survey is part of a program to study emission-line objects whose nature is not well established in the literature. In this paper we analyze SS73 56 (also known as Hen 3-1191, or simply Hen 1191) and [W74] 1 (also known as Hen 2-79). Previous investigations of stars from Sanduleak \& Stephenson's (1973) sample done by us led to an analysis of 33 emission-line stars (Pereira et al. 2003) and also led to analysis of a peculiar emission-line object, SS73 11, Landaberry et al. (2001) hereafter (L01).

SS73 56 was discovered as an emission-line object by Sanduleak \& Stephenson (1973), where it was classified as a $\mathrm{X}$ object, which means an object with a "Very-steep-Balmerdecrement". It was also catalogued by Henize (1976) having the number 1191 of his list, thus getting the name Hen 3-1191. Le Bertre et al. (1989) pointed that Hen 3-1191 might be a proto planetary-nebulae after this star has been observed during a infrared survey (Epchtein et al. 1987). It showed an energy distribution similar to that exhibited by other bright infrared source at $2.2 \mu \mathrm{m}$ (Hen 3-1379). A plot of the spectrum of SS73 56 (Hen 3-1191) between $5600 \AA$ and $9900 \AA$ is provided in the paper of Le Bertre et al. (1989) and several emission lines of single ionized iron may be recognized. In the ESO Catalogue

Send offprint requests to: C. B. Pereira, e-mail: claudio@on. br

* Based on observations made with the $1.52 \mathrm{~m}$ telescope at the European Southern Observatory (La Silla, Chile) under the agreement with the Observatório Nacional, Brazil. of Planetary Nebulae it was classified as possible planetarynebulae (Acker et al. 1992).

Hen 2-79 was discovered by Henize (1967) and was also present in the Weaver's search for T Tauri stars in the coalsack region (Weaver 1974). It was classified as a compact planetarynebulae by Allen \& Glass (1974), as a B star with forbidden emission by Allen et al. (1982) and as a peculiar emission-line star by Acker et al. (1992). Both stars studied in the present paper were classified as peculiar Be stars with infrared excess by Allen \& Swings (1976).

In this work we will analyze the emission-line spectra and discuss the nature of these two peculiar objects based on observations obtained by us. Whenever possible we will compare SS73 56 and Hen 2-79 with another peculiar object, namely SS73 11 (see L01). In Sect. 2 we present the data including: (i) the observations and the reduction procedure; (ii) a brief description of the spectra and (iii) an estimate of the extinction. In Sect. 3 we present some physical conditions of the object. The excitation temperature is derived using iron emission lines. In addition, the Self Absorption Curve (SAC) method (Friedjung \& Muratorio 1987) is employed to analyze the physical conditions of the iron-emitting region. In Sect. 4 we discuss the nature of the two objects and the conclusions are given in the last section.

\section{The data}

\subsection{Observations and reduction}

Spectroscopic observations were performed using a Boller \& Chivens spectrograph at the Cassegrain focus of the ESO $1.52 \mathrm{~m}$ telescope in La Silla (Chile) at March 4, 1999, 


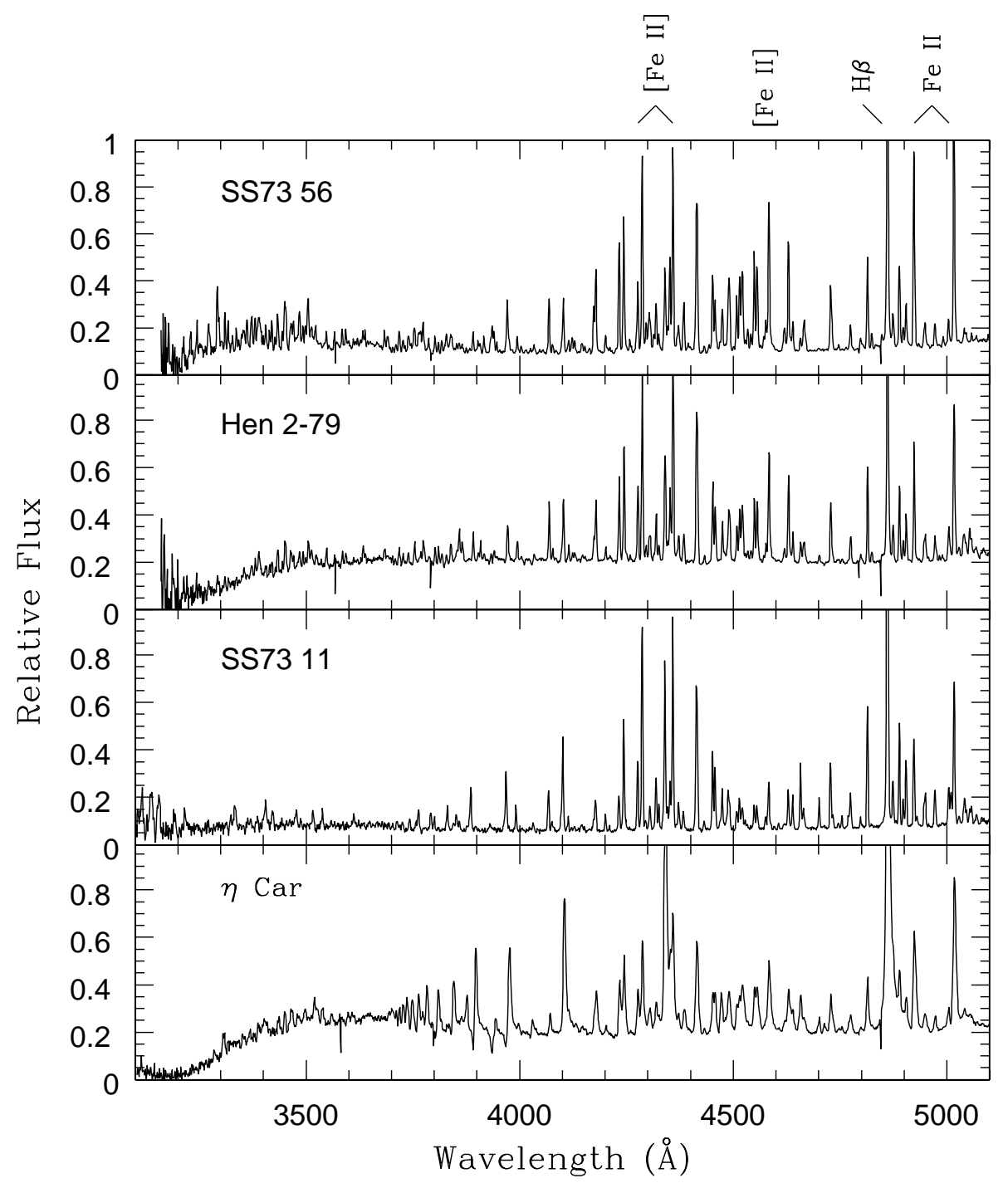

Fig. 1. Blue spectrum of SS73 56, Hen 2-79, SS73 11 and $\eta$ Car. Notice the presence of several emission lines of single ionized iron.

and June 13, 2001 (SS73 56) and February 3, 2001 and June 13, 2001 (Hen 2-79). A UV-flooded thinned Loral Lesser CCD \#39 $(2048 \times 2048,15 \mu \mathrm{m} /$ pixel) was used as the detector; it gives a high quantum efficiency in the blue and in the UV. Two instrumental setups were employed. The first made use of grating \#23 with $600 \mathrm{l} / \mathrm{mm}$, providing a resolution of $4.6 \AA$ in the range $\lambda \lambda 4000 \AA-8000 \AA$ and the other used the grating \#31 with $1200 \mathrm{l} / \mathrm{mm}$, resulting in a resolution of $1.9 \AA$ in the range $\lambda \lambda 3100 \AA-5100 \AA$. These setups have already been used to investigate other emission-line objects with the $1.52 \mathrm{~m}$ ESO telescope (Pereira et al. 2001; L01).

The spectra were reduced using standard IRAF tasks, from bias subtraction and flat-field correction, through spectral extraction and wavelength and flux calibration. Spectrophotometric standards from Oke (1974) and Hamuy et al. (1994) were observed. The slit orientation in the range (3200 $\AA-5100 \AA$ ) was aligned with the parallactic angle in order to minimize the light loss due to atmospheric refraction.

In the linearized spectra, the fluxes of emission lines have been measured by the conventional method adjusting a Gaussian function to the line profile thereby obtaining the intensity, the central wavelength and the line width at half power level. Uncertainties in the line intensities come mainly from the position of the underlying continuum. We estimate the errors in the fluxes to be about $20 \%$ for weaker lines (line fluxes $\approx 10$ on the scale of $\mathrm{H} \beta=100$ ) and about $10 \%$ for stronger lines.

SS73 56 and Hen 2-79 were also observed in high resolution mode, respectively on June 14, 2001 and February 9, 2001, with exposure times of $3600 \mathrm{~s}$ in both cases with FEROS spectrograph. The Fiber-Fed Extended Range Optical Spectrograph (Kaufer et al. 1999) is a bench-mounted Echelle spectrograph with the fibers located at the Cassegrain focus with a spectral resolution power of $R \approx 48000$. FEROS has a complete automatic on-line reduction.

\subsection{The spectra of SS7356 and Hen 2-79}

\subsubsection{Low resolution}

Our Cassegrain data of SS7356 and Hen 2-79 are displayed in Figs. 1 and 2. Figure 1 shows the spectrum in the $\lambda 3200-5100 \AA$ range, at a resolution of $1.9 \AA$ and Fig. 2 


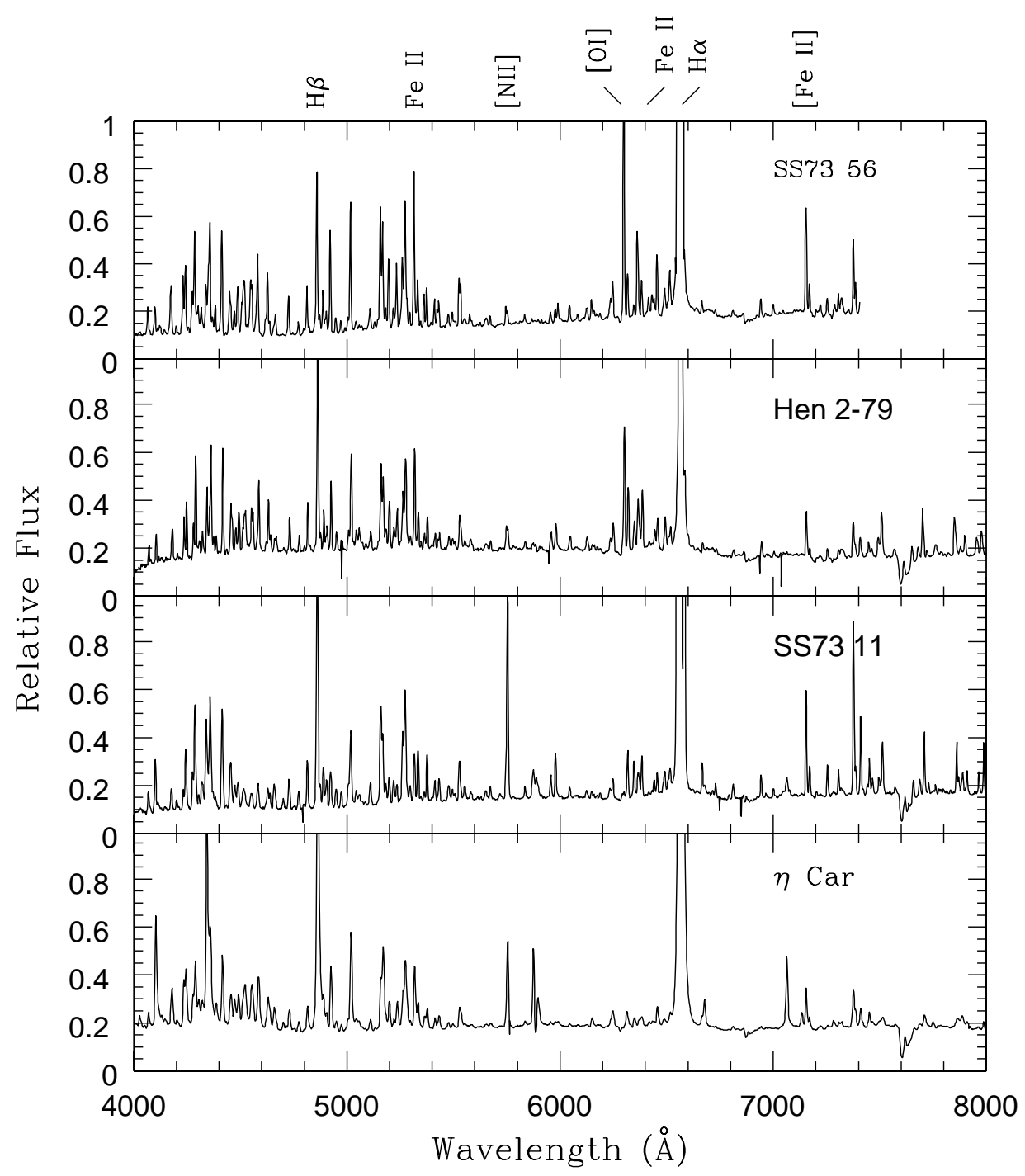

Fig. 2. Optical spectrum of SS73 56, Hen 2-79, SS73 11 and $\eta$ Car. Notice the strength of [O I] $6300 \AA$ in SS73 56 and in Hen 2-79 and the strengh of [N $\mathrm{II}] 5754 \AA$ in SS73 11 and in $\eta$ Car.

shows the $\lambda 3800-8000 \AA$ range at a resolution of $4.6 \AA$. We also plot the spectra of SS73 11 and $\eta$ Car for comparison.

The spectrum is completely dominated by several strong emission lines over a flat continuum, mostly due to single forbidden and permitted iron lines. The majority of the features are also present in the spectra of two other peculiar emission-line stars, $\eta$ Car and SS73 11 (L01). In particular, the iron spectra of the objects SS73 56, Hen 2-79 and SS73 11 look very similar in the blue range, $3100 \AA-5100 \AA$. On the other hand, the range $4000 \AA-8000 \AA$ reveals an important difference concerning the behavior of the transitions [O I] $6300 \AA$ and [N II] $5754 \AA$. The [OI] $6300 \AA$ feature is strong in SS73 56 and Hen 2-79 but weak in SS73 11 and almost undetectable in $\eta$ Car while the $\left[\mathrm{N}_{\text {II }}\right] 5754 \AA$ line is very weak in SS73 56 and Hen 2-79 but quite intense in SS73 11 and $\eta$ Car.

Columns 4 and 7 of Table 1 list the line flux, in units of $\mathrm{H} \beta=100$, of some multiplets of single ionized forbidden iron lines used for reddening and excitation temperature determination for SS73 56 and Hen 2-79. For line identifications we used the same procedure described in L01 which was applied to SS73 11. As was mentioned in the last paragraph, since the spectra of the two stars, SS73 56 and Hen 2-79 look very similar to SS73 11, most of the transitions are common in the three stars, regardless the relative intensities.

\subsubsection{High resolution}

The high-resolution spectra was used to better identify some features that are blended in the low resolution spectra. This procedure allowed us to estimate the different contributions of each transition. In addition, we used the high resolution data to better see the differences in strength of the oxygen forbidden line at $6300 \AA$ and the strength of the nitrogen forbidden line at $5754 \AA$. Figure 3 shows these three spectral regions for the three objects SS73 56, Hen 2-79 and SS73 11.

\subsection{Extinction}

We have determined the extinction parameter for SS73 56 and Hen 2-79 following the method proposed by Pagel (1969) as 
Table 1. Multiplets, wavelengths, excitation potential $(\chi)$, observed emission line fluxes $F(\lambda)$ in units of $\mathrm{H} \beta=100.0$, log $I$ (defined in the text) and the parameter $\beta$ (also defined in the text) of some selected [Fe II] emission lines used for reddening and excitation temperature determination of SS73 56 and Hen 2-79.

\begin{tabular}{|c|c|c|c|c|c|c|c|c|}
\hline & & & \multicolumn{3}{|c|}{ SS73 56 } & \multicolumn{3}{|c|}{ "Hen 2-79 } \\
\hline $\mathrm{M}$ & Identification & $\chi(\mathrm{eV})$ & $F(\lambda)$ & $\log I$ & $\beta$ & $F(\lambda)$ & $\log I$ & $\beta$ \\
\hline \multirow[t]{6}{*}{$4 \mathrm{~F}$} & 4639.68 & 2.77 & 3.9 & 0.12 & 4.29 & 6.2 & 0.32 & 4.48 \\
\hline & 4664.45 & 2.77 & 1.3 & 0.16 & 4.33 & - & - & - \\
\hline & 4728.07 & 2.69 & 11.7 & 0.27 & 4.48 & 10.3 & 0.21 & 4.42 \\
\hline & 4798.63 & 2.69 & 2.0 & 0.27 & 4.49 & - & - & - \\
\hline & 4889.63 & 2.57 & 17.3 & 0.33 & 4.62 & 12.3 & 0.18 & 4.46 \\
\hline & 5006.52 & 2.57 & 1.2 & 0.31 & 4.60 & - & - & - \\
\hline \multirow[t]{8}{*}{$6 \mathrm{~F}$} & 4382.75 & 2.82 & 1.6 & 0.08 & 4.30 & 3.3 & 0.40 & 4.70 \\
\hline & 4416.27 & 2.79 & 30.7 & 0.33 & 4.57 & 32.1 & 0.36 & 4.53 \\
\hline & 4432.45 & 2.83 & 2.0 & 0.33 & 4.54 & - & - & - \\
\hline & 4457.95 & 2.82 & 7.5 & 0.04 & 4.26 & 9.1 & 0.13 & 4.29 \\
\hline & 4488.85 & 2.82 & 4.2 & 0.20 & 4.42 & 4.9 & 0.27 & 4.43 \\
\hline & 4492.60 & 2.79 & 1.8 & 0.00 & 4.23 & 2.5 & 0.14 & 4.32 \\
\hline & 4514.90 & 2.82 & 2.4 & 0.19 & 4.41 & - & - & - \\
\hline & 4528.39 & 2.83 & 1.8 & 0.35 & 4.56 & - & - & - \\
\hline \multirow[t]{5}{*}{$7 \mathrm{~F}$} & 4287.40 & 2.88 & 46.0 & 0.38 & 4.58 & 43.2 & 0.36 & 4.49 \\
\hline & 4359.34 & 2.88 & 16.4 & 0.08 & 4.27 & 18.5 & 0.13 & 4.26 \\
\hline & 4413.78 & 2.88 & 18.6 & 0.28 & 4.48 & 21.8 & 0.35 & 4.49 \\
\hline & 4452.11 & 2.88 & 7.1 & 0.07 & 4.27 & 10.7 & 0.25 & 4.33 \\
\hline & 4474.91 & 2.88 & 3.1 & 0.01 & 4.21 & 4.5 & 0.17 & 4.32 \\
\hline \multirow[t]{4}{*}{$14 \mathrm{~F}$} & 7155.14 & 1.96 & 47.4 & 0.75 & 4.97 & 15.6 & 0.27 & 4.70 \\
\hline & 7171.98 & 2.01 & 13.1 & 0.75 & 4.93 & 6.2 & 0.42 & 4.82 \\
\hline & 7388.16 & 2.02 & 12.7 & 0.87 & 5.05 & 4.5 & 0.42 & 4.82 \\
\hline & 7452.50 & 2.02 & 13.4 & 0.75 & 4.93 & 4.1 & 0.23 & 4.63 \\
\hline $15 \mathrm{~F}$ & 6440.40 & 2.27 & 1.7 & 0.65 & 3.68 & - & - & - \\
\hline \multirow{5}{*}{$17 \mathrm{~F}$} & 5412.64 & 2.63 & 9.7 & 0.46 & 4.54 & 4.1 & 0.08 & 4.25 \\
\hline & 5495.82 & 2.63 & 5.1 & 0.47 & 4.55 & 2.5 & 0.17 & 4.32 \\
\hline & 5527.30 & 2.53 & 8.8 & 0.20 & 4.33 & 7.8 & 0.14 & 4.36 \\
\hline & 5654.85 & 2.53 & 2.4 & 0.60 & 4.73 & 0.8 & 0.11 & 4.34 \\
\hline & 5745.70 & 2.53 & 1.6 & 0.77 & 4.91 & - & - & - \\
\hline \multirow[t]{8}{*}{$18 \mathrm{~F}$} & 5107.96 & 2.77 & 4.3 & 0.51 & 4.56 & - & - & - \\
\hline & 5158.00 & 2.69 & 5.5 & 0.18 & 4.28 & 10.7 & 0.47 & 4.62 \\
\hline & 5181.97 & 2.77 & 2.8 & 0.18 & 4.23 & 4.1 & 0.34 & 4.45 \\
\hline & 5268.88 & 2.69 & 7.8 & 0.54 & 4.64 & 4.5 & 0.30 & 4.45 \\
\hline & 5273.38 & 2.57 & 21.2 & 0.45 & 4.61 & 12.8 & 0.23 & 4.44 \\
\hline & 5347.67 & 2.69 & 2.7 & 0.61 & 4.70 & 2.5 & 0.57 & 4.72 \\
\hline & 5433.15 & 2.67 & 12.2 & 0.62 & 4.90 & 5.8 & 0.47 & 4.64 \\
\hline & 5556.31 & 2.57 & 1.5 & 0.55 & 4.51 & 0.8 & 0.27 & 4.49 \\
\hline \multirow[t]{8}{*}{$19 \mathrm{~F}$} & 5111.63 & 2.65 & 5.6 & 0.16 & 4.29 & 4.1 & 0.02 & 4.20 \\
\hline & 5158.81 & 2.62 & 22.6 & 0.04 & 4.19 & 20.6 & 0.01 & 4.20 \\
\hline & 5184.80 & 2.68 & 1.1 & 0.33 & 4.44 & - & - & - \\
\hline & 5220.06 & 2.66 & & & & 3.3 & - & 4.15 \\
\hline & 5261.61 & 2.65 & 24.3 & 0.32 & 4.45 & 17.7 & 0.19 & 4.36 \\
\hline & 5296.84 & 2.68 & 3.9 & 0.25 & 4.37 & 2.9 & 0.12 & 4.28 \\
\hline & 5333.65 & 2.66 & 18.8 & 0.38 & 4.50 & 10.3 & 0.11 & 4.29 \\
\hline & 5376.47 & 2.68 & 18.0 & 0.47 & 4.58 & 10.0 & 0.21 & 4.37 \\
\hline \multirow[t]{9}{*}{$20 \mathrm{~F}$} & 4814.55 & 2.79 & 12.2 & 0.03 & 4.14 & 13.6 & 0.07 & 4.20 \\
\hline & 4874.49 & 2.83 & 5.1 & 0.27 & 4.36 & 4.1 & 0.18 & 4.28 \\
\hline & 4905.35 & 2.82 & 9.0 & 0.27 & 4.36 & 6.6 & 0.14 & 4.25 \\
\hline & 4947.38 & 2.79 & 2.4 & 0.24 & 4.34 & 2.5 & 0.26 & 4.38 \\
\hline & 4950.74 & 2.84 & 3.5 & 0.30 & 4.37 & 3.3 & 0.27 & 4.37 \\
\hline & 4973.39 & 2.83 & 4.7 & 0.33 & 4.41 & 3.3 & 0.18 & 4.28 \\
\hline & 5005.52 & 2.82 & 4.3 & 0.46 & 4.49 & 4.5 & 0.47 & 4.58 \\
\hline & 5020.24 & 2.84 & 2.4 & 0.12 & 4.19 & 3.7 & 0.30 & 4.40 \\
\hline & 5043.53 & 2.83 & 2.0 & 0.30 & 4.38 & 2.1 & 0.32 & 4.42 \\
\hline
\end{tabular}


Table 1. continued.

\begin{tabular}{|c|c|c|c|c|c|c|c|c|}
\hline \multirow[b]{2}{*}{$\bar{M}$} & \multirow[b]{2}{*}{ Identification } & \multirow[b]{2}{*}{$\chi(\mathrm{eV})$} & \multicolumn{3}{|c|}{ SS73 56} & \multicolumn{3}{|c|}{ Hen 2-79 } \\
\hline & & & $F(\lambda)$ & $\log I$ & $\beta$ & $F(\lambda)$ & $\log I$ & $\beta$ \\
\hline \multirow[t]{9}{*}{$21 \mathrm{~F}$} & 4243.98 & 3.14 & 12.2 & & & 11.5 & - & 3.71 \\
\hline & 4244.81 & 3.21 & 3.9 & 0.02 & 3.41 & 4.5 & 0.08 & 4.04 \\
\hline & 4276.83 & 3.19 & 13.7 & 0.04 & 3.45 & 12.5 & 0.01 & 3.97 \\
\hline & 4305.90 & 3.22 & 4.5 & 0.11 & 3.50 & 3.7 & 0.03 & 3.99 \\
\hline & 4319.62 & 3.21 & 9.8 & 0.10 & 3.49 & 9.1 & 0.07 & 4.03 \\
\hline & 4346.85 & 3.14 & 5.7 & 0.05 & 3.49 & 5.1 & 0.01 & 4.00 \\
\hline & 4352.78 & 3.19 & 7.3 & 0.09 & 3.50 & - & - & - \\
\hline & 4358.37 & 3.22 & 8.8 & 0.04 & 3.43 & 11.9 & 0.18 & 4.13 \\
\hline & 4372.43 & 3.21 & 5.7 & 0.15 & 3.55 & 4.8 & 0.08 & 4.03 \\
\hline \multirow[t]{3}{*}{$31 \mathrm{~F}$} & 6809.21 & 2.79 & 1.7 & 0.48 & 4.36 & 1.2 & 0.33 & 4.38 \\
\hline & 6966.32 & 2.84 & 0.6 & 0.50 & 4.29 & - & - & - \\
\hline & 7047.99 & 2.84 & 1.4 & 1.08 & 4.57 & - & - & - \\
\hline \multirow[t]{2}{*}{$34 \mathrm{~F}$} & 5477.25 & 3.32 & 3.0 & 0.43 & 3.86 & 2.5 & 0.35 & 4.12 \\
\hline & 5746.96 & 3.18 & 6.6 & 0.49 & 4.00 & 6.2 & 0.46 & 4.31 \\
\hline \multirow[t]{2}{*}{$35 \mathrm{~F}$} & 5163.94 & 3.37 & 4.7 & 0.16 & 3.89 & 4.5 & 0.14 & 3.92 \\
\hline & 5283.10 & 3.37 & 2.2 & 0.20 & 3.93 & 2.9 & 0.32 & 4.09 \\
\hline \multirow[t]{5}{*}{$39 \mathrm{~F}$} & 5551.53 & 3.89 & 1.7 & 0.44 & 3.79 & 1.6 & 0.62 & 3.86 \\
\hline & 5580.82 & 3.87 & 1.4 & 0.47 & 3.83 & 0.8 & 0.22 & 3.72 \\
\hline & 5613.27 & 3.87 & 1.4 & 0.60 & 3.96 & 0.8 & 0.35 & 3.81 \\
\hline & 5650.94 & 3.87 & 0.8 & 0.62 & 3.98 & - & - & - \\
\hline & 5718.20 & 3.87 & 0.3 & 0.10 & 3.46 & - & - & - \\
\hline $43 \mathrm{~F}$ & 6944.91 & 3.80 & 1.7 & 0.66 & 3.86 & - & - & - \\
\hline \multirow[t]{2}{*}{$44 \mathrm{~F}$} & 6188.55 & 3.95 & 1.7 & 0.52 & 4.36 & - & - & - \\
\hline & 6473.86 & 3.93 & 1.3 & 0.85 & 4.39 & - & - & - \\
\hline a2D-b2D & 6353.10 & 4.50 & 0.8 & 0.54 & 3.40 & - & - & - \\
\hline \multirow[t]{2}{*}{ a2G-a2I } & 5870.00 & 4.10 & 1.7 & 0.30 & 3.46 & - & - & - \\
\hline & 6044.10 & 4.08 & 1.5 & 0.43 & 3.59 & - & - & - \\
\hline a4H-a2I & 7975.30 & 4.50 & 6.1 & 0.52 & 4.23 & 7.4 & 0.61 & 4.54 \\
\hline \multirow[t]{2}{*}{ a2G-b2D } & 4898.61 & 4.50 & 3.5 & 0.36 & 3.51 & 4.1 & 0.43 & 3.60 \\
\hline & 5060.08 & 4.74 & 2.7 & 0.55 & 3.71 & 2.5 & 0.52 & 3.69 \\
\hline a2G-c2D & 4576.40 & 4.10 & 4.1 & 0.95 & 4.07 & 2.9 & 0.79 & 3.88 \\
\hline \multirow[t]{2}{*}{ a2G-c2G } & 5673.22 & 4.10 & 3.0 & 0.36 & 3.57 & 2.9 & 0.33 & 3.66 \\
\hline & 5835.44 & 4.10 & 3.6 & 0.50 & 3.72 & 2.9 & 0.41 & 3.73 \\
\hline \multirow[t]{2}{*}{ a2P-b2D } & 5627.25 & 4.70 & 1.7 & 1.13 & 4.03 & - & - & - \\
\hline & 5799.00 & 4.70 & 0.8 & 1.09 & 3.99 & - & - & - \\
\hline a2P-c2D & 5048.18 & 4.70 & 2.7 & 0.67 & 3.71 & 2.9 & 0.70 & 3.75 \\
\hline
\end{tabular}

previously applied to SS7311 (L01). We first measured the observed line fluxes of some forbidden lines (those with excitation potential between 2.5 and $3.2 \mathrm{Ev}$ ) between $4100 \AA$ and $5500 \AA$. We then plot $\log I$ (defined below) in the ordinates against the reciprocal wavelength $(1 / \lambda(\mu \mathrm{m}))$ in the abscissa. The ordinate $\log I$ is the difference between the logarithm of the observed flux and the logarithm of the emitted flux by the source in the same wavelength range and is defined by the relation

$\log I=\log \left(F_{\text {obs }}(\lambda)\right)-(\log (5000 / \lambda)+\log (g A)-0.56 \chi+2.0)(1)$

where $F_{\text {obs }}(\lambda)$ is the observed line flux in units of $\mathrm{H} \beta=100$, $g A$ is the statistical weight multiplied by the transition probabilities and $\chi$ is the excitation potential of the upper level of the transition. The factor 2.0 accounts for the $\log F(\mathrm{H} \beta)=100$.

The 5th and 8th columns of Table 1 give $\log I$ as defined in the text for both stars. The value of $E(B-V)$ is obtained multiplying the difference of the values of the ordinates corresponding to the $B$ and $V$-bands by -2.5 . The color excess $E(B-V)$ which results from this procedure is $0.68 \pm 0.33$ for SS73 56 and $0.30 \pm 0.14$ for Hen 2-79.

\section{Physical conditions}

\subsection{Excitation temperature}

The excitation temperature was determined following Viotti (1969) which again was applied to SS73 11 (L01). Since in the spectra of SS73 56 and Hen 2-79 there are several forbidden emission lines of single ionized iron it is possible to derive the excitation temperature in the emitting region. Columns 6 and 9 of Table 1 give the parameter $\beta$, defined as $\beta=$ $\log \left(F_{\mathrm{c}}(\lambda) \lambda(\AA) / g A\right)$, for SS73 56 and Hen 2-79, while Col. 3 shows the excitation potential of the forbidden lines used in this calculation. In the above expression $F_{\mathrm{c}}(\lambda)$ is the line intensity 

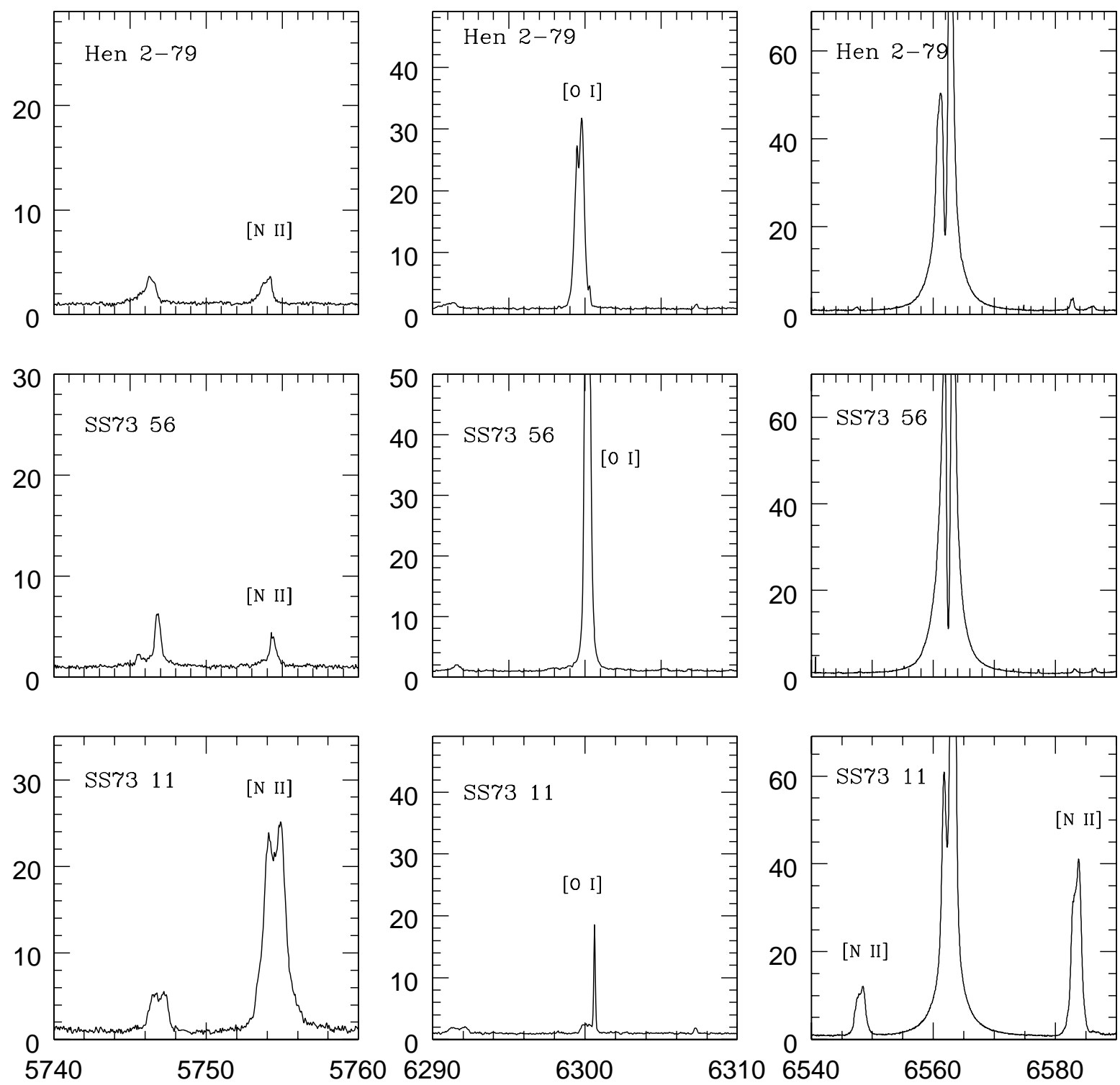

Fig. 3. Relative Flux versus Wavelength $(\AA)$ of the high resolution spectra obtained with Feros spectrograph in the regions around the forbidden nitrogen line at $5754 \AA$, the forbidden oxygen line at $6300 \AA$ and around $\mathrm{H} \alpha$ and [N $\mathrm{N}_{\text {II }}$ 6548, $6589 \AA$ lines for the two stars analyzed in this work and SS73 11.

in units of $\mathrm{H} \beta=100$ corrected for reddening and $\lambda(\AA)$ is given in Angstroms. The obtained temperature based on this method is $T_{\text {exc }}=11000 \pm 1200 \mathrm{~K}$ for SS73 56 and $T_{\text {exc }}=11500 \pm$ $800 \mathrm{~K}$ for Hen 2-79.

\subsection{The SAC method}

The self-absorption curve method was developed by Friedjung \& Muratorio (1987) and re-explained by Kastner (1999). It is an empirical way to determine several physical parameters of an emission line source by ploting normalized flux, obtained from the measured line intensities versus the respective optical thickness. Self absorption gives a linear growth of the logarithm of the normalized flux in the higher opacity region. On the side of the low opacity, the ordinates become constant. The coordinates of the region where the slope changes, gives the information on the column density of the respective ion.
We applied the method to both SS7356 and Hen 2-79 that present several permitted and forbidden lines of Fe II, similarly to SS73 11 (L01). The selection of the multiplets made here was also described in L01.

Figure 4 shows the plots, for SS73 56 and Hen 2-79, $\log g f \lambda$ versus $\log F(\lambda) \lambda^{3} / g f$, where $F(\lambda)$ is the line Flux in cgs units, $\lambda$ is the wavelength and $g f$ is the oscillator strength of the transition. Each point represents one transition line, for the selected permitted transitions. Points representing the permitted transitions are linearly correlated with negative slope nearly equal to -1.0 , along the optically thick region of the curve. Points corresponding to forbidden transitions were reduced to one per multiplet, with coordinates equal to the averages of the multiplet components. Our data of permitted lines do not allow us to see the bending of the SAC curve, but the point representing the forbidden lines give constraints to the location where the thin case is attained. The points where the straight 


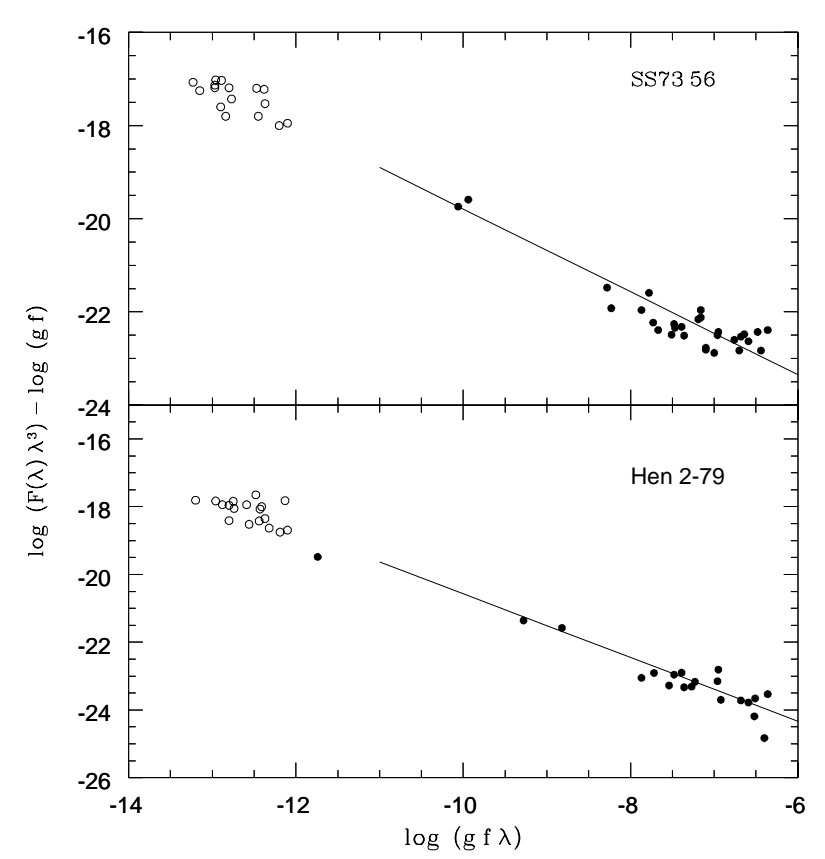

Fig. 4. Empirical Self-Absorption Curve of SS73 56 and Hen 2-79 derived from $\mathrm{Fe}$ II multiplet overlapping. Filled circles correspond to permitted transitions (multiplets 27, 29, 37, 38, 48 and 49) and open circles points to forbidden transitions.

line line bend to the horizontal thin case branch, just below the clouds of the forbidden lines are $X_{0}=-11.5$ and $Y_{0} \approx-18.75$ for SS73 56 and $X_{0}=-11.5$ and $Y_{0} \approx-19.5$ for Hen 2-79.

$X_{0}$ provides the value of the Fe II column density of the ground term divided by the characteristic line broadening velocity $v_{0}$, which we assume to be the thermal one. We found: $\log N_{0} / g_{0} \approx 18$ for SS73 56 and Hen 2-79 and from that value, if all the iron is in the form of Fe II and the iron abundance is the cosmic one, the hydrogen column density is of the order of $10^{25} \mathrm{~cm}^{-2}$ for SS73 56 and Hen 2-79.

\section{The Nature of SS7356 and Hen 2-79}

The most remarkable aspect of the spectra of SS7356 and Hen 2-79 is their similarity to the spectrum of SS73 11 and to that of the well known LBV $\eta$ Car (see Figs. 1 and 2). Moreover, all of them except $\eta$ Car exhibit several characteristics of the "B[e]-phenomenon" (Lamers et al. 1998, hereafter L98). We will roughly follow the same discussion as we did for SS73 11 (L01), in order to try to group SS73 56 and Hen 2-79 in some known category among peculiar emission stars.

Again, the major impediment to address the true natures of SS73 56 and Hen 2-79 is the absence of any estimate of their distances, which implies that their luminosities are also unknown. In this case, these objects could be in principle categorized in any of the following groups: supergiant $\mathrm{B}[\mathrm{e}]$, premain sequence B[e] ("HAeB[e] star") or compact planetary nebulae. Several Fe II emission lines are seen in the spectrum of HAeB[e] stars (see for instance the spectrum of V 380 Ori in L98). On the other hand SS73 56 and Hen 2-79 are not associated with star-forming regions and their spectra do not show inverse P-Cygni profiles. Hence they are not likely to be in the class of "HAeB[e]" objects.

SS73 56 was supposed to be a planetary-nebulae by Acker et al. (1992). Perhaps the main reason for that was the flat continuum and the presence of numerous emission lines. However, due to the absence of lines of higher excitation such as [O III] and [Ne III] this object was rejected as planetary nebulae. We have also compared our data with some compact planetary nebulae ("cPNB[e]-stars") according to the list given in Table 3 of L98. The group listed in that table have several stars whose spectra differ from each other. As an example, $\mathrm{Hb} 12$, M 1-26 and Mz 3 are planetary nebulae with spectra, respectively, WN7, Of and O9.5/B0 (Acker et al. 1992). The nature of V 704 Cen is still uncertain; it might be symbiotic (Pereira et al. 1998). Hen 2-139 is a symbiotic star (Allen 1984). Any of the two stars studied in this paper display some spectroscopic characteristics of the stars listed in Table 3 of L98.

On the basis of the spectral characteristics of SS73 56 and Hen 2-79 we cannot exclude the possibility that these objects are massive stars in advanced evolutionary stages. However it is difficult to confirm (or discard) such a scenario without an accurate determination of their luminosities. If this is the case, they could be B[e] Supergiant or Luminous Blue Variable candidates. Again, it would not be easy to point one type or another. Nota et al. (1996) and Morris et al. (1996) presented evidences of several spectral similarities among B[e]Sgs and LBVs. Schulte-Ladbeck et al. (1993) had previously reported a connection but they suggested that the presence of a circunstellar disk may be frequent in $\mathrm{B}[\mathrm{e}]$ Supergiants in order to explain the intrinsic polarization observed in some cases. Zickgraf (1989) claimed that it is possible to use [N II] and $\left[\mathrm{O}_{\mathrm{I}}\right]$ lines to distinguish a $\mathrm{B}[\mathrm{e}] \mathrm{Sg}$ from a LBV. SchulteLadbeck (1998) referred to this criteria in a work on a evolutionary status among massive stars. According to it is more probable that SS73 56 and Hen 2-79 are B[e] Supergiants than LBVs, since their oxygen forbidden lines at $6300 \AA$ are quite strong while their nitrogen forbidden lines at $5754 \AA$ are rather weak. The opposite behavior is seen in SS73 11 and in $\eta$ Car (see Figs. 1-3). Anyway, a more precise conclusion requires other data such as polarization, UV lines and distance estimates.

Another possibility for SS7356 and Hen 2-79 would be that these two objects were proto planetary-nebulae candidates, given the similarity to the spectrum of Hen 401 (García-Lario et al. 1999). There are other two arguments which favour the proto planetary-nebulae candidate classification, e.g. the infrared colors of SS73 56 as well as its imaging presented in Le Bertre et al. (1989). In addition, both SS73 56 and Hen 401 have basically the same bipolar morphology seen in some other well-known proto planetary-nebulae such as M2-9 (García-Lario et al. 1999). There are no infrared colors or imaging of Hen 2-79, but considering the spectrum similarities between Hen 2-79 and SS73 56 we suggest the same nature for these two objects. The infrared excess as given by the IRAS flux ratio for the two objects mentioned above through the Fig. 1 of Pottasch et al. (1988), place them in a region occupied by the $\mathrm{OH} / \mathrm{IR}$-stars. It is worth mentioning that the presence of oxygen lines in the spectra of SS73 56 and in Hen 2-79 
can be reconciled with their positions in the IRAS flux ratio diagram. This point was also stressed by Le Bertre et al. (1989) in a discussion of Hen 1191 (=SS73 56).

The spectra of SS73 56 and Hen 2-79 have some similarity with two hot post-AGB stars presented by Parthasarathy et al. (2000), i.e., MWC 930 and MWC 939. The spectra of these two stars in the wavelength $\lambda \lambda$ 3800-5020 $\AA$ are shown in Vijapurkar et al. (1998). Although the spectral region cover a short wavelength range, several iron lines are seen in their spectra. If MWC 930 and 939 have the same spectral characteristics when observed in another spectral region beyond $5000 \AA$ this would indicate that MWC 930 and 939 could also be proto planetary-nebulae candidates.

\section{Summary and conclusions}

We have analyzed the low- and high-resolution optical spectra of two peculiar emission-line objects SS73 56 and Hen 2-79. From a set of several forbidden and permitted emission lines of single ionized iron, we derived some physical conditions in the envelope, such as excitation temperature, electron temperature and density. The SAC method was used to investigate the physics of the single ionized iron region. Finally we discussed the nature of the studied objects comparing them with other peculiar emission-line objects already analyzed in the literature. The similarity of SS73 56 and Hen 2-79 to SS73 11 and $\eta$ Car spectra is remarkable. However, it should be also stressed that this similarity is mainly related to the single ionized lines. The great difference is the strength of [O I $6300 \AA$ in respect to [N II]5754 $\AA$ in SS73 56 and Hen 2-79 against SS73 11. The infrared colors as well as the optical imaging of SS73 56, which is similar to Hen 401, and the spectral characteristics (which are also similar to Hen 401) indicate that the two objects may be considered proto-planetary nebulae candidates.

Acknowledgements. We thank the referee, Dr. Hans van Winckel, for valuable remarks and comments.

\section{References}

Acker, A., Ochsenbein, F., Stenholm, B., et al. 1992, Strasbourg-ESO Catalogue of Planetary Nebulae

Allen, D. A. 1984, PASA, 5, 369
Allen, D. A., \& Glass, I. S. 1974, MNRAS, 167, 331

Allen, D. A., \& Swings, J. P. 1976, A\&A, 47, 293

Allen, D. A., Baines, D. W. T., Blades, J. C., \& Whittet, D. C. B. 1982, MNRAS, 199, 1017

Epchtein, N., Le Bertre, T., Lepine, J. R. D., et al. 1987, A\&AS, 71, 39

Friedjung, M., \& Muratorio, G. 1987, A\&A, 85, 233

García-Lario, P., Riera, A., \& Manchado, A. 1999, ApJ, 526, 854

Kastner, S. O. 1999, A\&A, 351, 1016

Kaufer, A., Stahl, S., Tubbesing, S., et al. 1999, The Messanger, 95, 8

Hamuy, M., Suntzeff, N. B., Heathcote, S. R., et al. 1994, PASP, 106, 566

Henize, K. 1967, ApJS, 14, 125

Henize, K. 1976, ApJS, 30, 491

Lamers, H. J. G. L. M., Zickgraf, F. J., de Winter, D., Houziaux, L., \& Zorec, J. 1998, A\&A, 240, 117 (L98)

Landaberry, S. J. C., Pereira, C. B., \& de Araújo, F. X. 2001, A\&A, 376, 917 (L01)

Le Bertre, T., Epchtein, N., Gouiffes, C., Heydari-Malayeri, M., \& Perrier, C. 1989, A\&A, 225, 417

Morris, P. W., Eenens, P. R. J., \& Blum, R. D. 1996, ApJ, 470, 597

Nota, A., Pasqualli, A., Drissen, L., et al. 1996, ApJS, 102, 383

Oke, J. B. 1974, ApJS, 27, 21

Pagel, B. E. J. 1969, Nature, 221, 325

Parthasarathy, M., Vijapurkar, J., \& Drilling, J. S. 2000, A\&AS, 145, 269

Pereira, C. B., Landaberry, S. J. C., \& Conceição, F. 1998, AJ, 116, 1971

Pereira, C. B., Schiavon, R. P., de Araújo, F. X., \& Landaberry, S. J. C. 2001, AJ, 121, 1071

Pereira, C. B., Franco, C. S., \& de Araújo, F. X. 2003, A\&A, 397, 927

Pottasch, S. R., Bignell, C., Olling, R., \& Zilstra, A. A. 1988, A\&A, 205, 248

Sanduleak, N., \& Stephenson, C. B. 1973, ApJ, 185, 899

Schulte-Ladbeck, R. E., Leitherer, C., Clayton, G. C., et al. 1993, ApJ, 407, 723

Schulte-Ladbeck, R. 1998, in Proc. of the Paris Workshop on B[e] stars, ed. A.-M. Huvert, \& C. Jaschek (Kluwer Academic Press), 253

Vijapurkar, J., Parthasarathy, M., \& Drilling, J. S. 1998, Bull. Astr. Soc. India, 26, 497

Viotti, R. 1969, Astrophys. Spa. Sci, 5, 323

Weaver, W. B. 1974, ApJ, 189, 263

Zickgraf, F. J. 1989, in Angular Momentum and Mass Loss for Hot Stars, ed. R. A. Wilson, \& R. Stalio (Kluwer Academic Publishers), 245 